\title{
Relation between breast milk energy density and the pre-conception BMI from donations of a human milk bank
}

\author{
Yanira Flórez Matías ${ }^{1}$, Liliana Ladino Melendez ${ }^{2}$ and Sandra Patricia Guevara Núñez ${ }^{1}$ \\ ${ }^{1}$ Universidad Nacional de Colombia, Bogotá, Colombia and \\ ${ }^{2}$ Universidad El Bosque, Bogotá, Colombia
}

\section{Abstract}

Human breast milk is a high value biological substance that grants numerous benefits both to mother and child. Many aspects can produce changes in the milk composition; among them, the pre-conception body mass index (Pre-conception BMI) which is included as a factor that can influence the fat levels and therefore, the milk's energy density. To identify if there is a relation between the breast milk energy density from a human milk bank and Preconception BMI. It is a retrospective study that used information from donations registered in the human milk database at Hospital de Kennedy during 2016. The dependent variable is the energy density from 3 kinds of milk: colostrum, mature and transition milk. As independent variable, Preconception BMI was taken. The R free program version 3.3.0 was used for statistical analysis. The energy density average found for the 3 kinds of milk were: $20.59+/-2.3 \mathrm{kcal} / \mathrm{oz}$, $20.51+/-2.17 \mathrm{kcal} / \mathrm{oz}$ y $19.21+/-2.53$ for colostrum, transition milk and mature milk respectively. The Preconception BMI average from the donors was $23.56 \mathrm{Kg} / \mathrm{m}^{2}$ for colostrum, $23.9 \mathrm{Kg} / \mathrm{m}^{2}$ for transition milk and $23.1 \mathrm{Kg} / \mathrm{m}^{2}$ for mature milk. No relation between Preconception BMI and energy density of colostrum was found. However, for transition milk and Preconception BMI a directly proportional relation was found $(\mathrm{p}<0.01)$, and for mature milk and Preconception BMI an inversely proportional relation was determined $(p=0.0387)$. There are internal and external factors than can affect the breast milk lipid content, such as movements of reserves through the organism to protect the milk production, hormonal action, dietary intake, physical activity and socio-economic and cultural aspects; that is why it is not possible to analyze the preconception BMI as a unique predictor of breast milk energy density in an isolated manner. Preconception BMI is related to human breast transition and mature milk energy density in donations registered in 2016 at Kennedy Hospital.

\section{Conflict of Interest}

There is no conflict of interest 